INPLASY PROTOCOL

To cite: Tang et al. Evaluation of the clinical efficacy of stem cell transplantation in the treatment of spinal cord injury: systematic review and metaanalysis. Inplasy protocol 202140034. doi: 10.37766/inplasy2021.4.0034

Received: 06 April 2021

Published: 07 April 2021

Corresponding author: Qiaorui Tang

tangqr@mails.jlu.edu.cn

Author Affiliation: Jilin University

Support: China National Natural Found.

Review Stage at time of this submission: Data analysis.

Conflicts of interest:

None declared.

\section{Evaluation of the clinical efficacy of stem cell transplantation in the treatment of spinal cord injury: systematic review and meta-analysis}

Tang, Q1; Xue, H²; Zhang, Q3; Guo, Y4; Xu, H5; Liu, Y6; Liu, J7.

Review question / Objective: This meta-analysis aims to provide a comprehensive reference for treating SCI with stem cell transplantation, for example which cell types, which methods and how many cells are good for patients. We also analyzed the urodynamic index and adverse reactions to provide further evidence.

Condition being studied: Spinal cord injury (SCl) is a grievous neurological disease caused by traumatic and non-traumatic injuries that leads to different degrees of sensorimotor injury and sphincter dysfunction. The incidence of $\mathrm{SCl}$ is high and increases annually. Meanwhile, the healthcare cost is extremely high. $\mathrm{SCl}$ is still incurable because of high disability, and there is no suitable therapy to improve functional recovery. Currently, The clinical efficacy of these therapies, which are applied for $\mathrm{SCl}$, are not satisfactory. Stem cells have a great application prospect due to the ability to renew themselves and differentiate into functional cells. In recent years, stem cells are frequently used in basic experimental research and clinical studies for SCI. These stem cells aim to deliver growth factors, provide trophic support, improve the micro-environment, modulate the inflammatory response, and remyelinate. All of them can live within the host spinal cord for a period of time and promote functional recovery through differentiating into neurons and glial cells. However, there are inconsistencies in efficacy of clinical trials.

INPLASY registration number: This protocol was registered with the International Platform of Registered Systematic Review and Meta-Analysis Protocols (INPLASY) on 07 April 2021 and was last updated on 07 April 2021 (registration number INPLASY202140034).

\section{INTRODUCTION}

Review question / Objective: This metaanalysis aims to provide a comprehensive reference for treating $\mathrm{SCl}$ with stem cell transplantation, for example which cell types, which methods and how many cells are good for patients. We also analyzed the 
urodynamic index and adverse reactions to provide further evidence.

Condition being studied: Spinal cord injury (SCl) is a grievous neurological disease caused by traumatic and non-traumatic injuries that leads to different degrees of sensorimotor injury and sphincter dysfunction. The incidence of $\mathrm{SCl}$ is high and increases annually. Meanwhile, the healthcare cost is extremely high . SCl is still incurable because of high disability, and there is no suitable therapy to improve functional recovery. Currently, The clinical efficacy of these therapies, which are applied for $\mathrm{SCl}$, are not satisfactory. Stem cells have a great application prospect due to the ability to renew themselves and differentiate into functional cells. In recent years, stem cells are frequently used in basic experimental research and clinical studies for SCl. These stem cells aim to deliver growth factors, provide trophic support, improve the micro-environment, modulate the inflammatory response, and remyelinate. All of them can live within the host spinal cord for a period of time and promote functional recovery through differentiating into neurons and glial cells. However, there are inconsistencies in efficacy of clinical trials.

\section{METHODS}

Participant or population: Patients with spinal cord injury.

Intervention: Stem cell transplantation.

Comparator: Surgery, rehabilitation or physiotherapy, medication.

Study designs to be included: Clinical control trials or randomized controlled trials.

Eligibility criteria: 1. Study subjects: patients with SCI; 2. Intervention: treatment with stem cell transplantation; 3. Outcome indicators: (1) sensory and motor function indicators: ASIA Impairment Scale (AIS) grading; (2) urodynamic indices: maximum urinary flow rate, maximum bladder, residual urine volume, maximum detrusor pressure and ISCIS score scale; 4. Study types: clinical control trials (CCTs) or randomized controlled trials (RCTs).

Information sources: Electronic databases.

Main outcome(s): Sensory and motor function indicator (AIS improvement rate); Urodynamic index; Adverse event.

Additional outcome(s): The correlation between the incidence of neuropathic pain and treatment measures.

Quality assessment / Risk of bias analysis: The Cochrane manual was used to assess the quality of the included studies. The evaluation items are as follows: 1. random sequence generation; 2 . allocation concealment; 3 . blinding of participates and personnel; 4 . blinding of outcome assessment; 5. incomplete outcome data; 6. selective reporting; 7 . other bias. According to the extracted information, each item in the quality evaluation of the included studies had three levels: "low risk of bias", "unclear risk of bias" or "high risk of bias".

Strategy of data synthesis: For sensory and motor function indicators, dichotomous data were assessed using ORs with 95\% confidence intervals $(95 \% \mathrm{Cls})$ and $p$ values. The chi-square test or 12 test was used to evaluate heterogeneity. 120.1 was interpreted as low heterogeneity, and a fixed effects model was used; $12>50 \%$ or p $<0.1$ was interpreted as high heterogeneity, and a random effects model was used. For the urodynamic index and adverse reactions, a systematic review was conducted due to a small number of studies and inconsistent data.

Subgroup analysis: Subgroup analyses (before treatment; cell types; cell numbers; transplantation methods).

Sensitivity analysis: There is no sensitivity analysis.

Country(ies) involved: China. 
Keywords: spinal cord injury; stem cell transplantation; meta-analysis; AIS grading; Urodynamic index.

Contributions of each author:

Author 1 - Qiaorui Tang.

Author 2 - Hui Xue.

Author 3 - Qiao Zhang.

Author 4 - Ying Guo.

Author 5 - Hao Xu.

Author 6 - Ying Liu.

Author 7 - Jiamei Liu. 